\title{
Analysis of the Situation Semantics Solution to Paradoxes
}

\author{
Li Dai $^{1}$, Ji-hua Zhang ${ }^{2}$
}

${ }^{1)}$ School of Literature and Chinese as a Foreign Language, Mianyang Normal University, Mianyang, Sichuan, China

${ }^{2)}$ Department of Science and technology, Mianyang Normal University, Mianyang, Sichuan, China

Abstract-As the important model in the research of semantic paradoxes in recent years of the West, the situation semantics solution to paradoxes develops a school of its own among many programs for semantic paradoxes resolution. This paper attempts to clarify and summarize systematically the basic ideas and spiritual essence of the solution, to discuss the solution's promotion to many related theories.

Keywords — semantic paradox, program for paradox resolution, situation semantics

\section{情境语义学解悖方案探析 \\ 代利 ${ }^{1}$ 张继华 ${ }^{2}$ \\ 1) 绵阳师范学院文学与对外汉语学院, 绵阳, 四川, 中国 2) 绵阳师范学院科技处, 绵阳, 四川, 中国}

摘 要 情境语义学解悖方案是近年西方语义悖论研究的重要典范, 在诸多语义悖论解决方案中独树一帜。本文试图系统梳理总结 这一方案的基本理路与精神实质, 并评析这一方案对相关理论发展的推动。

关键词 语义悖论, 解悖方案, 情境语义学

\section{1. 引言}

语义悖论问题既是逻辑史上古老的难题之一，也是当 代逻辑哲学中的热点问题。语义悖论的解决不仅对逻辑学 基础的完善与发展有重大意义, 同时对语言学、哲学、计 算机和人工智能科学的研究也有极其重要的影响。

自从说谎者悖论出现以来, 围绕它产生了很多解悖方 案, 但大多数方案并不令人满意。情境语义学是二十世纪 八十年代初出现的一种新的语义理论。1985 年, 美国学者 巴威斯 (Jon Barwise) 和埃切曼迪 (John Etchemendy) 把 情境语义学试用于解决语义悖论, 后来取得较大成功。巴 威斯和埃切曼迪的解悖方案可以简称为“情境语义学解悖 方案”, 即运用情境语义学并通过奥斯汀阐释的形式而对悖 论做出诊断和说明。从情境语义学的角度来考察语义悖论 的解决, 开辟了语义悖论研究的新理路。

\section{2. 情境语义学解悖方案的基本理路}

2.1 “情境”概念的引入

“情境”既是情境语义学的核心概念, 也是情境语义学 解悖方案得以建立的重要基石。什么是“情境”呢？在情境 语义学看来, “情境是由主体选择或区分的高度组织起来的 世界的一部分。”[1]简单地说, 由认知主体认知的某一或某 些个体, 在某一时空条件下, 具有某种性质或处于某种关 系中, 就构成一个情境。情境由个体、关系 (性质)、时空 单位等基本要素构成。例如, “昨天下午五点钟, 小张和小 李聊天。”这个句子描述了这样一个情境——时间: 昨天下 午五点钟; 两个个体: 小张和小李; 个体处于的关系: 聊 天。这个句子没有涉及事件发生的地点。由此可见, 情境 与语境“貌离神合”, 只是在作形式刻画时才能将它们严格 区分。“情境”概念具有两个重要特点: 第一, 客观性, 即 情境是现实世界的一部分。情境语义学认为, 在理解自然 语言的意义时, 仅参照现实世界的有限部分就足够了, 不

本文为四川省教育厅科研项目“现代逻辑视域下的群体理性聚合研究” (13SB0159)及绵阳师范学院引进人才科研基金资助项目“基于判 断聚合逻辑的群体理性条件研究”(QD2012B03)的阶段性成果之一 
仅没有必要, 而且也不可能将整个现实世界都考虑在内。 情境只是现实世界的一个有限组成部分, 而不是它的全部。 情境语义学不设定最大情境。第二, 主观性, 即情境与认 知主体相关。情境中的世界不再是纯客观的世界, 而是由 主体区分、挑选的现实世界的一部分。人们在区分、挑选 世界上的个体、性质、关系时, 必然要受到自己的目的、 信念等主观因素的影响。在整个世界中, 究竟什么能够组 成一个情境, 什么不能够组成一个情境, 这是与所讨论的 主体密切相关的。

\section{2 “奥斯汀型命题观”的提出}

在深受牛津日常语言学派主要代表奥斯汀的影响下, 巴威斯和埃切曼迪提出了与“罗素型命题观”根本不同的一 种新型命题观一一 “奥斯汀型命题观”, 从而为语义悖论的 消解提供了一个全新视角。

巴威斯和埃切曼迪指出, 他们之所以使用“罗素型命题 观”这个称谓, 并不是因为罗素有什么独特的命题理论, 而 是因为罗素的观点是经典观点的典型代表。其实, “罗素型 命题”就是经典命题理论中的“命题”概念。

罗素认为, 命题是对某一事实加以肯定或否定的直陈 句。什么是事实呢? 罗素指出, “当我说一个事实时, 我指 的不是世界上的一个简单事物, 我指的是某物具有某种性 质或某些事物具有某种关系。例如, 我不把拿破仑称作事 实, 而是把他有野心或者他娶了约瑟芬称作事实。” [2]可 见, 在罗素看来, 一个事实 (原子事实) 所描述的是, 某 物具有某种性质或某些事物之间具有某种关系; 一个原子 命题就是, 对某物具有某种性质或某些事物之间具有某种 关系的肯定或否定。罗素认为, 命题和事实是同构的, 它 们之间存在着严格的对应关系。与原子命题相对应的是原 子事实, 与由原子命题组合而成的分子命题相对应的是分 子事实, 与普遍命题相对应的是普遍事实。

需要强调的是, 罗素型命题只是对“事态”或“事态集合” 的直接描述，在其中没有“情境”或“语境”的要素。“事态” 是指特定时空单位中个体 (对象) 具有某性质或个体（对 象) 间具有某关系, 现实事态就是事实。这是否说明持有 罗素型命题观的学者并不重视语境呢? 答案是否定的。持 有罗素型命题观的学者, 在分析自然语言语句时, 大多坚 持“语境惟一确定原则”, 即只有在确定的语境中, 才能确 定语句的意义, 从而才能把握语句所表达的命题。然而, 他们只是把语境原则作为识别命题的途径, 他们所理解的 语境是外在于命题的。只要给定语境, 就能确定语句所表 达的命题, 而且一个语句在给定语境中只表达惟一一个命 题。可见, 罗素型命题观建立的是语句与命题的一一对应
关系。其实, 自然语言中的同一语句, 在不同情境中, 可 以表达不同的命题。正是看到了罗素型命题观的局限性, 巴威斯和埃切曼迪提出了把情境作为命题内在要素之一的 “奥斯汀型命题观”, 从而为语义悖论的消解提供了一个全 新视角。

奥斯汀在讨论“真理”概念时指出, 日常语言与世界相 联的方式乃经由两种约定: 描述约定与指示约定。描述约 定是指把语句普型与某些情境类型相关联; 指示约定是指 把语句殊型与世界上的历史-现实情境相关联。[3]这里的 “情境类型”是反映不同情境之间共性的概念。例如, 两名 篮球运动员, 在不同的时空条件下, 都分别投进了一个球。 这两个事件所涉及的具体情境虽然不同, 但二者之间亦具 有一定共性（在某一情境中, 某个人投进了一个球)。“情 境类型”揭示的就是这种共性。巴威斯、埃切曼迪对奥斯汀 的上述观点作了如下重述: “依据奥斯汀, 一个合法的陈述 A 提供两个东西: 一个历史的（或实际的）情境 SA, 和一 个情境类型 TA。前者是现实世界的某个有限部分：说话者 使用奥斯汀所谓“指示约定”去指称它; 而后者大致说来就是 依据与 (自然) 语言相关联的“描述约定”, 由陈述所确定的 情境属性。”[4]虽然奥斯汀本人未使用“命题”这一术语, 但 是巴威斯、埃切曼迪认为: 由于命题就是陈述所表达的思 想内容, 因而他们所命名的“奥斯汀型命题”也应包括, 陈 述所在的实际情境以及陈述所描述的情境类型。也就是说, 奥斯汀型命题要本质地包含情境因素。显然, 一个奥斯汀 型命题应由三个要素构成: 一个自然语言的语句普型、一 个对该语句普型的索引的和指示的元素的外延指派、一个 该命题所处世界的部分模型。在这种刻画下, 真值谓词本 身不是索引的, 但“真的”在一个命题中的一次出现, 是依 据在相关情境中该谓词的部分外延赋值的。这样, 一个奥

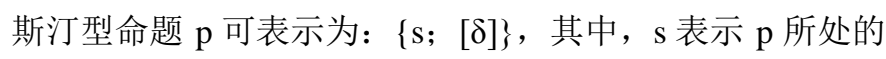
情境, $\delta$ 表示 $\mathrm{p}$ 所描述的事态, [ $\delta$ ]表示取决于 $\delta$ 的情境类型。 令 $\mathrm{p}$ 作为强化的说谎者语句“本语句不是真的”之缩写, 依 据明显的经验事实可确定 (用双引号表示语句的名称): “p 不是真的”等同于 $\mathrm{p}$, 故强化的说谎者语句可记作 “ $\mathrm{p}: \mathrm{p}$ 不 是真的”。设强化的说谎者语句“ $p: p$ 不是真的”所在的情境 为 $s$, 则根据奥斯汀型命题观, $p$ 在 $s$ 中所表达的命题 $\mathrm{fs}$ 断 定的是, $\mathrm{fs}$ 不是真的这一语义事实是 $s$ 中的一个事实 (某事 态在情境 $\mathrm{s}$ 中实现叫做 $\mathrm{s}$ 中的事实); $\mathrm{p}$ 在 $\mathrm{s}$ 中所表达的命题 可表示为: $f s=\{s ;[T r, f s ; 0]\}$, 其中, “Tr,fs; 0 ”表示命题 fs 描述的事态, 即 fs 不是真的 ( “fs 为真”赋值为 0 ) 这一语 义事实。单独讨论时, fs 所描述的这一事态可记作 $<\mathrm{Tr}$, fs; $0>$ 。

综上所述, 一个罗素型命题可以简单地记作 $[\delta]$, 而一 个奥斯汀型命题应记作 $\{\mathrm{s},[\delta]\}$ 。按照罗素型命题观, 情境 
是外在于命题的现实世界的组成部分, 而按照奥斯汀型命 题观, 情境是内在于命题的, 是命题的一个不可或缺的重 要组成部分, 同一语句在不同情境中所表达的命题各不相 同。

\section{3 “奥斯汀型真值观”的确立}

在对于 “真”的认识中, 符合论的观点可谓历史悠久。 罗素型命题观与奥斯汀型命题观对于 “命题”认识的不同, 也决定了两种真值观——“罗素型真值观”与 “奥斯汀型真 值观”之差异, 虽然这两种真值观同属符合论的范畴。

亚里土多德指出, “凡以不是为是、是为不是者这就是 假, 凡以实为实、以假为假者, 这就是真。”[5]罗素对“真” 之认识, 与亚里土多德的思想可谓一脉相承。罗素指出, “如 果我说“现在正在下雨”, 那么, 在某种天气条件下, 这句话 是真的, 而在另一种天气条件下, 这句话是假的, 这种使 我的这句话成真成假的天气条件, 就是我所说的“事实”。”[6] 可见, 在罗素看来, 命题的真假取决于命题是否与事实相 符合。罗素认为, 命题与事实是同构的: 复合命题由原子 命题构成, 原子命题由名称构成; 与之相对应, 事实由事 态构成, 事态表现为对象的排列组合。当人们谈论原子命 题的真假时, 主要谈论的是它所描述的事态之真假。如果 一原子命题所描述的事态在客观世界中为真, 则此命题就 为真, 否则为假。同理, 分子命题之真假依据于原子事实 的集合之真假。

显然, 按照罗素型真值观, 命题的真是客观世界中的 事实使之为真, 而命题之所以为假, 是由于客观世界中不 存在使之为真的事实。其实, 客观世界中不存在某一事实 而使一命题为真, 这并不能说明此命题一定为假, 即不能 断定一命题为真, 并不一定能够断定它为假。同时, 罗素 型真值观以客观世界作为参照物来描述命题的真, 也过于 抽象、庞大。正是出于对罗素型真值观的不满, 巴威斯、 埃切曼迪在奥斯汀思想之影响下, 以奥斯汀型命题观为基 础, 提出了一种新的真值观一一奥斯汀型真值观”, 从而 为合理地消解语义悖论奠定了坚实基础。

以奥斯汀型命题观为基础, 巴威斯、埃切曼迪对命题 的真值问题进行了重新考察。其中, 奥斯汀对陈述之真的 认识, 也同样对巴威斯、埃切曼迪产生了较大影响。

奥斯汀指出, “一个陈述, 如果指示约定使它与之相关 联的历史事态(它“指称”的那个事态) 是这样一种类型的事 态: 描述约定使作出陈述时使用的语句与该类事态相关联, 那么我们就说这个陈述是真的。”[3]巴威斯、埃切曼迪对此 也作了如下重述: 依据奥斯汀, 一个合法陈述 A 提供两个 方面: 一个历史的（或实际的）情境 SA, 和一个情境类型
$\mathrm{TA}$ 。如果 $\mathrm{SA}$ 具有类型 TA, 则 $\mathrm{A}$ 为真, 否则为假。[4]基于 上述认识, 巴威斯、埃切曼迪指出: 对于一个可表示为 $\{\mathrm{s}$; $[\delta]\}$ 的奥斯汀型命题 $\mathrm{p}, \mathrm{p}$ 为真, 当且仅当, 事态 $\delta$ 属于情 境 $\mathrm{S}$ 。

由此看来, 情境不仅是构成奥斯汀型命题的一个不可 或缺的重要部分, 而且还是刻画奥斯汀型命题之真值的一 个重要参量。当相关情境发生变化时, 一个语句所表达的 命题就会发生相应变化, 同时命题的真值亦随之而变。由 此看来, 罗素型命题观与奥斯汀型命题观有着根本区别: 前者以客观世界作为确定命题真值的参照物, 而后者用以 确定命题真值的参照物却是客观世界的一部分——相关情 境。

\section{4 对强化的说谎者悖论的消解}

在诸多语义悖论中, 说谎者悖论可以说是最古老、最 富有代表性的。近年来, 说谎者悖论的变体强化的说谎者 悖论, 已成为检验一种新的解悖方案是否成功的试金石。 不少学者认为, 只要消解了强化的说谎者悖论, 其他语义 悖论问题便可迎刃而解。出于对伯奇方案视真值谓词为索 引谓词的不满, 巴威斯、埃切曼迪将情境语义学理论试用 于解决语义悖论问题, 通过对命题及其真值的重新审视, 成功地实现了对强化的说谎者悖论的消解。其消解过程如 下:

证明: （1）强化的说谎者语句在情境 $\mathrm{s}$ 中所表达的 命题 fs(在 $U$ 中) 为假。

设 $U$ 为客观世界的整体模型（total model）, 认知主体 A 表达强化的说谎者语句“p: $\mathrm{p}$ 不是真的”相关的实际情境 为 $\mathrm{s}$, 则有 $\mathrm{s} \subseteq \mathrm{U}$ 。根据奥斯汀型命题观, $\mathrm{p}$ 在情境 $\mathrm{s}$ 中所表 达的命题应为: $f s=\{s ;[\mathrm{Tr}, \mathrm{fs} ; 0]\}$ 。

(1) 假设 fs 在 $s$ 中为真, 则 $\mathrm{fs}$ 所描述的事态应属于 $\mathrm{s}$, 即 $\langle\mathrm{Tr}$, fs; $0>\in \mathrm{s}$;

(2) 据有关模型的特征: $\langle T r, p ; 1\rangle \in U$ 当且仅当 $\mathrm{p}$ 是真 的, 这样, 如果 $\mathrm{fs}$ 在 $\mathrm{s}$ 中真, 则有 $\langle\mathrm{Tr}, \mathrm{fs} ; 1>\in \mathrm{s}$;

(3) 因为 $\mathrm{s}$ 是模型 $\mathrm{U}$ 中一个实际情境, 且据有关模型的 特性, 一个模型不能既包括一个事态又包括这个事态的否 定, 而(1)和(2)却表明: 两个相互矛盾的事态同时出现在模 型 $U$ 中的一个实际情境 $s$ 中, 这是不可能的。因此, fs 在 $U$ 中假。

注意, 这里的结论是 fs 在“U”中假而不是在“s”中假。 为什么呢? 下面的证明表明, fs 为假的相关实际情境已不 再是 $\mathrm{s}$ 。

（2）fs 为假的相关实际情境必不是 S。

证明：(1) 假设 $\mathrm{fs}$ 为假的相关实际情境是 $\mathrm{s}$, 则 $<\mathrm{Tr}$, fs; 
$0>\in \mathrm{s} ;$

(2) 由于 $\langle\mathrm{Tr}, \mathrm{fs} ; 0>$ 正是 $\mathrm{fs}$ 所描述的事态, 因此, $\mathrm{fs}$ 在 $\mathrm{s}$ 中真, 即 $<\mathrm{Tr}, \mathrm{fs} ; 1>\in \mathrm{s}$;

(3) 根据(1)和(2), 两个相互矛盾的事态同时出现在模型 $\mathrm{U}$ 中的一个实际情境 $\mathrm{s}$ 中, 这是不可能的。因此, fs 为假的 相关实际情境必不是 $\mathrm{S}$ 。[7]

综合 (1) 和 (2) 可以看出, 当某人在情境 $s$ 中说“我 说的这句话不是真的” 时, 他一定是在表达一个假命题, 但 此命题为假这一语义事实却不能在相同的情境 $\mathrm{s}$ 中被断言。 当然, 我们可以设想 $\mathrm{fs}$ 为假这一语义事实包含在另一情境 $\mathrm{s}^{\prime}$ 之中。也就是说, 强化的说谎者语句所表达的命题为真 和为假的情境是不同的, 因而就不能构成矛盾, 悖论被自 然消解了。这就如同, 一个人在中国说““现在是早上八点钟” 为真”, 而另一个人同时在美国说“ “现在是早上八点钟'为 假”, 其实二者并没有真正的矛盾, 因为它们是不同情境中 的“真”和“假”。

\section{3. 情境语义学解悖方案的重要启示}

语义悖论的根源问题是解决语义悖论的首要问题。对 语义悖论根源的认识不同, 相应的解悖方案也随之不同。 已往诸多语义悖论解决方案的缺陷表明, 人们还没有正确 地认识语义悖论的根源。情境语义学解悖方案对语义悖论 的合理解决启示我们, 语义悖论的真正根源在于对于相关 情境的忽略。

从情境语义学解悖方案对以强化的说谎者悖论为代表 的语义悖论的消解来看, “情境”概念的引入在其中起着关 键作用。“情境”概念的引入, 使得同一语句所表达的命题, 在不同的情境中, 可以具有不同的真值。强化的说谎者语 句所表达的命题在其所在的情境中只能为假, 而其为真只 能是在另外不同情境中为真。因为这里的“真”和“假”已属于 不同情境中的“真”和“假”, 因而并不构成矛盾。

情境语义学解悖方案正确确立了语义悖论的研究重 点, 对语句所表达的命题作了深入思考, 对 “真”等语义概
念作了重新审视, 进而合理地解决了语义悖论问题。同时 这也表明, 经典逻辑对语义概念的刻画存在很大缺陷。

经典逻辑使用的是人工语言, 它在处理语句时, 只考 虑语句的外延, 即真值。这样, 用经典逻辑处理自然语言 的意义时会遇到很多难题。例如, 如果仅从真值角度考虑, 所有语句只能划归为两类: 真和假。如此处理直接导致的 后果是, 所有逻辑上等值的语句都具有相同的语义解释, 这显然与自然语言的实际不符。其实, 自然语言中的绝大 多数语句是不能一成不变地被划分为真或假的。同一语句 可能在有些情境中为真, 而在另外一些情境中又为假。可 以说, 正是意识到经典逻辑在研究和处理自然语言丰富意 义方面的不足, 巴威斯等人才决定要创建一种适用于处理 自然语言丰富意义的语义理论。在深受言语行为理论的影 响下, 巴威斯和佩里提出了情境语义学这一新颖的语义学 理论。

\section{参考文献(References)}

[1] K. Devlin, Logic and Information, Cambridge University Press, 1991, p.30.

[2] J. Tu, Review of the Contemporary Western Philosophers, vol. 1, p. $68,1996$.

[3] J. L. Austin, "Truth", Proceedings of the Aristotelian Society, Supp.Vol.xxiv, 1950.

[4] J. Barwise and J. Etchemendy, The Liar: An Essay on Truth and Circularity, Oxford University Press, 1987, pp.28-29.

[5] Aristotle, Metaphysics, Trans. by Shoupeng $\mathrm{Wu}$, The Commercial Press, p. 79, 1959.

[6] Russell, Logic and knowledge, Trans. by Lijun Yuan, The Commercial Press, p. 182, 1996.

[7] J. Wang, "The Complementarity of Logic in the View of the Situation Semantics Solution”, Economist, 7, 2006. 